\title{
Dietary glycemia as a determinant of health and longevity
}

Elizabeth A. Whitcomb, Chung-Jung Chiu, and Allen Taylor

Laboratory for Nutrition and Vision Research, USDA-Human Nutrition Research Center on Aging at Tufts University. Boston MA 02111

The role of diet in extending lifespan and healthspan has been the subject of much research and debate. Our recent epidemiological and in vivo data suggest that carbohydrate quality can be a major determinant in prolonging eye health. Additionally, excessive carbohydrate intake can contribute to the exacerbation of many different diseases. The metabolic diversity of the tissues that are affected by excessive carbohydrate intake suggests that dietary carbohydrate quality may affect cellular homeostasis.

Key words: glycemic index, glycation, ubiquitin, proteasome, autophagy, eye disease

\section{1-Introduction and definition of glycemic index}

\subsection{Introduction}

Extending healthful life is a millennia-old dream and objective. During the intervening centuries a multitude of concoctions and remedies have been offered, usually with few substantiated results. During the last century it was demonstrated that limiting caloric intake is associated with extended life in many mammals, albeit results remain to be clarified in humans (Colman et al., 2009; Mattison et al., 2012; McCay et al., 1935; McCay and Crowell, 1934; Walford and Crew, 1989; Walford, 1986;

Weindruch, 1984, 1991, 1996). A myriad of modeling studies have revealed signaling pathways that are associated with life extension and the last two decades have seen an interest in the types of dietary carbohydrates that might confer health advantage, and possibly longevity. Loss of vision due to agerelated cataracts or age-related macular degeneration is widely prevalent, affecting about $85 \%$ and $15 \%$ of the elderly respectively. With centenarians among the fastest growing segments of societies, and with loss of vision a very costly personal and societal burden, there is keen interest in extending vision- that is, delaying age-related macular degeneration and cataract- or diminishing risk for these debilities. Using extensive epidemiologic and nutritional information from the Nurses' Health Study and AgeRelated Eye Disease Study (AREDS) we determined that measures of total carbohydrate, and even more so, glycemic index (GI), are associated with visual health (Chiu, 2011; Chiu et al., 2006b, 2007a; Chiu et al., 2007b, c; Weikel, 2011; Weikel et al., 2012a). We also modeled this relationship in mice in order to elucidate etiologic relationships between dietary glycemia, visual health, and genetics (Rowan et al., 2014; Uchiki et al., 2012). 


\subsection{Definition of glycemic index}

The glycemic index (GI) of a food describes how quickly carbohydrates in our food are converted into blood glucose. GI is defined as the rise in blood glucose within a two hour period elicited by a portion of food containing $50 \mathrm{~g}$ of available carbohydrate, relative to the rise elicited by $50 \mathrm{~g}$ of glucose (See Fig. 1 for example). Dietary glucose or high GI foods increase blood glucose rapidly and lead to a compensatory postprandial hypoglycemia (green and red lines in Fig. 1) whereas a low Gl food increases blood glucose to a lower extent (blue line in Fig. 1). Two foods can have identical total carbohydrate content and be isocaloric, but have different $\mathrm{Gl}$, based on how quickly the carbohydrates are converted to glucose. Recent studies indicate that the types or quality of carbohydrate foods consumed by an individual have a major impact on health. It has been shown that relative to consuming higher GI diets, consuming lower $\mathrm{GI}$ diets is associated with attenuated blood glucose increases, lower postprandial hypoglycemia, lower hyperlipidemia, lower insulinemia, less elevation of inflammatory markers, and less counter regulatory endocrine responses (Jenkins et al., 2002; Ludwig, 2002; Scribner et al., 2008). The typical diet in the United States is high glycemic, suggesting that many chronic diseases in the US may be ameliorated by dietary changes (Chiu, 2011).

\section{2-Glycemic Index, disease and proteopoise.}

Throughout this work proteostasis is referred to as proteopoise to indicate that there is a dynamic rather than static relationship between proteins, insults and the cellular machinery that must recognize and selectively remove the damaged proteins.

\subsection{Risk of age related macular degeneration (AMD)}

Work in our lab has focused on relationships between the risk for age-related eye diseases, primarily age-related macular degeneration (AMD) or cataract and consuming high GI diets. AMD is the leading cause of irreversible blindness in the elderly. There are two types of AMD: "wet AMD" results from neovascularization in the choroid of the retina, while "dry AMD" is correlated with the appearance of drusen, which are white or yellow deposits of extracellular material that accumulate between the Bruch's membrane and the retinal pigment epithelium (RPE). Dry AMD is the most prevalent type representing nearly $90 \%$ of all AMD cases. While there are several treatments which target the neovascularization in wet $A M D$, currently, there are no treatments to delay or reverse dry AMD. Both types of AMD result in death of the photoreceptor cells and vision loss. Late stage dry AMD is correlated with the loss of large areas of RPE and photoreceptors known as geographic atrophy.

Our epidemiological data indicate that consumption of high GI diets is associated with increased prevalence and increased progression of AMD (Fig. 2) (Chiu et al., 2006a; Chiu et al., 2007a; Chiu et al., 2007b); even when diets contain the same amount of total carbohydrate. Persons in the highest quintile of GI intake had increased risks of large drusen, neovascularization and geographic atrophy as compared to the lowest quintile (Chiu et al., 2007a). Work from other groups confirms that high GI diets increase risk of AMD including soft drusen (Kaushik et al., 2008). These studies suggest that changes in diet could diminish the risk of developing AMD and progression of the disease. The risks of AMD associated with 
smoking (Smith et al., 2001) and genetic variations (Fritsche et al., 2013) are within similar ranges to the risks associated with the consumption of high GI diets (Fig.2).

Many other age-related diseases are also exacerbated by high GI diets: individuals consuming high GI diets are at increased risk for type II diabetes, cardiovascular disease as well as cataracts (Chiu, 2011). Since cataract results from post-synthetic modifications of lens proteins, called crystallins, it would appear that elevated levels of sugars might be involved in cataractogenesis as well. These relationships are intriguing because the lens is an avascular, anoxic environment with what is probably the slowest metabolism in the body. Right next to it is the retina, the most oxygenated and highly vascularized tissue in the body. The metabolic diversity of the tissues that are adversely affected by high Gl carbohydrate intake suggests that the relationship between dietary carbohydrate quality and cellular homeostasis is of fundamental physiological importance and, perhaps, that there is a common etiologic link between dietary glycemia and the various diseases.

\subsection{Accumulation of advanced glycation endproducts (AGEs)}

Chronic hyperglycemia, such as would be expected with consuming a high GI diet, can lead to glycoxidation or glycation, which is a non-enzymatic reaction of sugars and their metabolites with proteins. These modifications are known as advanced glycation end products (AGEs). AGEs accumulate in tissues during aging in both animals and humans. Shown in Figure $3 \mathrm{~A}$, using an antibody which recognizes a particular AGE modification (MG-H3 (Wang et al., 2015)), AGEs accumulate in human lenses and the mouse brain upon aging (Uchiki et al., 2012). We speculated that AGE accumulation would be exacerbated by long term consumption of high GI diets. Indeed, we found increased AGEs in mice fed high GI diets (Fig. 3B) as compared to age-matched controls that consumed the lower GI diet. Furthermore, animals fed high GI diets are also at increased risk for metabolic changes similar to prediabetes such as impaired glucose tolerance ((Rowan et al., 2014; Uchiki et al., 2012) and unpublished data). These animals also have increased retinal lesions consistent with an AMD like phenotype (Uchiki et al., 2012; Weikel et al., 2012b). Retinal changes such as increased basal laminar deposits and decreased basal infoldings were observed in mice fed high GI diets. Basal laminar deposits have also been observed in humans with AMD (Biesemeier et al., 2014). A recent publication indicates that consumption of high AGE containing foods leads to metabolic syndrome and impaired cognitive function (Cai et al., 2014) suggesting that AGEs in the diet are damaging in vivo.

Although fructose is, by definition, a low $\mathrm{Gl}$ sugar, diets high in fructose are also associated with impaired health (Lim et al., 2010; Lustig, 2013). One possible mechanism may be that fructose is able to form AGEs at an increased rate compared to glucose (Bousova et al., 2011; Sadowska-Bartosz et al., 2014). Thus, the toxicity of fructose may be due to its ability to form AGEs. However, fructose in vivo is no more glycating than glucose in a yeast model (Semchyshyn et al., 2014) and a recent study suggests that fructose by itself is not correlated with impaired health that can't be attributed to increased overall calorie intake (Chung et al., 2014). Thus, it is unclear if the glycating activity of fructose is more damaging in vivo than glucose. Given the abundance of fructose in our diets, the relationship between fructose consumption and AMD risk deserves additional attention. 


\subsection{In vitro analysis of proteopoise and AGEs}

We hypothesize that increased accumulation of AGE-modified proteins leads to long term disruption of cellular homeostasis and decreased overall health. Adequate identification and removal of damaged proteins is necessary to maintain proteopoise. Several proteolytic pathways can dispose of unwanted proteins when they are damaged or no longer required.

The proteasome is a multisubunit protease which degrades proteins which are unfolded or damaged (Dudek et al., 2005; Marques et al., 2006; Shang et al., 2001; Zhang et al., 2007) and which need to be removed rapidly. This includes many cell cycle regulatory proteins (Buschhorn and Peters, 2006; Peters, 2002; Yew, 2001). The proteasome can degrade proteins that are ubiquitinated. The ubiquitin pathway adds the small protein ubiquitin to substrates via a relay of three different families of enzymes. The multiple ubiquitin enzymes allow exquisite substrate specificity in directing degradation (Pickart and Fushman, 2004). Thus, damaged proteins can be identified and targeted for proteasomal degradation by the addition of ubiquitin moieties. When the pathway is working properly, a substrate can attain very high mass as it becomes multiubiquitinated. If these high mass ubiquitinated substrates are not degraded, they may aggregate and become insoluble. Prior to degradation, the ubiquitin is removed and recycled, while the substrate goes on to degradation into peptides.

The lysosome degrades proteins that come from the extracellular environment through endocytosis, as well as intracellular proteins that are delivered via autophagy. Autophagy was initially identified as a pathway which could recycle bulk cellular proteins under conditions of nutrient deprivation (Cuervo and Dice, 1998; Mortimore et al., 1988). Autophagy can also degrade whole organelles and can exhibit substrate specificity (Tolkovsky, 2009; Wong et al., 2012). Both proteasome and autophagic/lysosomal proteolytic capacities decrease during aging (Cuervo and Dice, 2000; Pereira et al., 2003), thus potentially contributing to the accumulation of damaged proteins.

We posited that accumulation of AGEs during aging and consumption of high GI diets leads to disruption of protein homeostasis; this in turn may contribute to the development of amyloid or protein conformational diseases including cataracts and AMD. To elucidate the effects of AGE accumulation on protein homeostasis, we developed an in vitro system. Methylgyloxal (MGO) is a highly reactive primary glucose metabolite. Short term exposures to MGO in vitro mimic long term exposures to glucose and its metabolites in vivo. Treatment of RPE cells in culture with MGO resulted in detectable levels of AGEs after 2 hours of exposure (Fig. 4, compare lanes 1 and 2). Timely removal of damaged proteins is essential to preserve health. Thus, we asked which intracellular proteolytic pathways were involved in the clearance of AGEs. Using specific inhibitors to the proteasome, autophagic, and lysosomal pathways, we determined that all of these pathways are involved in the clearance of AGE modified proteins (Fig. $4 \mathrm{~A})$. Cells were treated with MGO for $2 \mathrm{~h}$ and then allowed to clear the AGE-modified substrates in the absence of MGO. Over the $20 \mathrm{~h}$ chase time, the amount of AGEs decreased to $70 \%$ of the starting amount (compare lanes 2, 3 and 7). The proteasome inhibitor epoxomicin delayed clearance of the AGEs, showing nearly twice the levels of AGEs as the control cells without inhibitor at the 20h time point. Importantly, epoxomicin treatment retarded the clearance of very high mass species, which are unable to enter the resolving gel (see lanes 4 and 8 ). Because ubiquitination results in very high mass 
proteins, any perturbation in the deubiquitination process, proteasome inhibition or crosslinking of the moieties due to oxidative or glycative insults would also allow the build-up of very high mass ubiquitin conjugated proteins that may be cytotoxic. These data indicate that AGEs can be cleared by the ubiquitin proteasome pathway (see (Uchiki et al., 2012) for further evidence and discussion). Additional studies indicate activities within the ubiquitin proteasome pathway are compromised due to glycative stress (Uchiki et al., 2012).

We also tested whether inhibitors of the lysosome and autophagic pathway affected clearance of AGEs. Chloroquine, which raises intralysosomal $\mathrm{pH}$, thereby inhibiting lysosomal proteases, decreases clearance of AGEs (compare lanes 3 to 5 and 7 to 9). These results suggest that the lysosome is involved in the clearance of AGE-modified substrates. Note we also observe very high mass ubiquitinated and AGE-modified protein accumulating upon chloroquine treatment (lane 5), suggesting that some ubiquitinated AGE-modified proteins may be degraded via the lysosome. These data agree with recent literature indicating that the lysosomal and proteasomal pathways cooperate to degrade damaged proteins (Park and Cuervo, 2013). Further data indicated that whereas the glycated proteins arrive at the lysosome, they seem to accumulate at the inner membrane rather than reaching the proteolytic machinery within the lysosome (Uchiki et al., 2012). As such, they may exacerbate disruption of proteopoise by diminishing proteolytic capacity. These observations were corroborated when we examined overall proteolytic turnover in RPE cells treated with MGO; MGO treatment decreased proteolytic turnover over 50\% (Fig. 4B). These data suggest that long term hyperglycemia leads to decreased proteolysis.

To determine specifically if the autophagic pathway was involved in clearance of AGEs, we used the autophagy inhibitor 3-methyl adenine (3MA). 3MA treatment also decreased clearance of AGE-modified proteins (compare lanes 3 to 6 and 7 to 10). Collectively, our results indicate that both the proteasome and autophagic proteolytic pathways are employed to clear AGE modified proteins.

\section{3-Model and conclusions}

During aging and stress, the efficacy of proteolytic systems decreases (Cuervo and Dice, 2000; Pereira et al., 2003; Taylor, 2012). This leads to increased accumulation and aggregation of damaged proteins and toxicity due to the accumulation of aggregated proteins (Figs. 3A and 5). Protein precipitation diseases such as cataract and Alzheimer's disease are increased in the aged, likely exacerbated by the decreased proteolytic capacities observed in aging (Morimoto and Cuervo, 2014). Our recent data indicate that glycemic stress also leads to the accumulation of damaged and modified proteins. Additionally, glycemic stress decreases proteolytic turnover (Fig. 4B, Fig. 5) leading to decreased turnover of damaged proteins. We also observe the accumulation of high mass glycated proteins when the ubiquitin and lysosomal pathways are inhibited (Fig. 4). High mass moieties are often insoluble: in cataract, insoluble aggregated proteins interfere with the passage of light. Thus, in aged lenses, or in lenses of persons consuming high GI diets, the accumulation of high mass AGE-modified proteins may contribute to cataractogenesis. We hypothesize that there is a vicious cycle of diet and metabolism-induced glycative stress, accumulation of AGEs, and attenuated cell and tissue protein homeostasis and dynamics. Thus, 
long term consumption of high GI diets may contribute to decreased proteopoise and exacerbate a number of age-related protein precipitation diseases including AMD and cataract. 
Vitae:

Elizabeth A. Whitcomb Ph. D. is a scientist at the Human Nutrition Research Center on Aging at Tufts University. Her work focuses on the role of the ubiquitin pathway in controlling the fate of substrates involved in cell division, migration, and differentiation.

Chung-Jung Chiu D.D.S. Ph.D. is a scientist at the Human Nutrition Research Center on Aging at Tufts University and an assistant professor in the Department of Ophthalmology at Tufts Medical Center. His research interests include age-related eye diseases and their environmental, genetic, and nutritional risk factors, prediction and pathogeneses.

Allen Taylor Ph.D. is the Director of the Laboratory for Nutrition and Vision Research (LNVR). Studies in the LNVR focus on the intersection of aging, proteolysis, epidemiology, metabolomics, microbiomics, and nutrition, particularly as applied to age related vision disorders.

Acknowledgements: This material is based upon work supported by the U.S. Department of Agriculture - Agricultural Research Service (ARS), under Agreement No. 58-1950-4-003. Additional funding from the National Eye Institute to AT EY-13250 EY-21212. We also thank Dr. Sheldon Rowan for work on Figure 2. 
Figure 1: Changes in blood glucose after consumption of high and low glycemic foods. Change in blood glucose in $\mathrm{mmol} / \mathrm{L}$ is plotted versus time in minutes.

Figure 2: Risk of developing AMD. Odds or risk ratios of developing AMD due to consumption of high glycemic index diets (first 7 points- (Chiu et al., 2006a; Chiu et al., 2007b; Kaushik et al., 2008)) smoking (Smith et al., 2001) or variants in ARMS2 or CFH genes (Fritsche et al., 2013). The center points (red) are odds or risk ratios, while the lines show the upper and lower confidence intervals.

Figure 3: AGEs accumulate during aging and upon consumption of high GI diets. A) Human lenses were obtained from cadavers from young (age 22) or old (age 76) donors. Lenses were harvested from young (age months) and old (age months) mice. AGEs were detected using anti-MG-H1 as described (Uchiki et al., 2012). Recent evidence indicates that this antibody actually recognizes the isomer MG-H3 (Wang et al., 2015) and we have labeled them as such. Ponceau, total protein staining (human samples) or GAPDH expression was used for quantification. B) Samples of the same aged mice: brain, lens, retina and liver from mice fed low or high glycemic index diets for 10 months.

Figure 4: MGO treatment results in AGE accumulation and decreased protein degradation. A) RPE cells were treated with $1 \mathrm{mM} \mathrm{MGO}$ for $2 \mathrm{~h}$. MGO was then removed and cells were incubated in the presence or absence of inhibitors for times indicated. AGEs were detected by anti-MG-H3 (Wang et al., 2015). B) RPE cells were labeled with ${ }^{3} \mathrm{H}$-Tyr for 60 hours and then cells were treated with MGO for 2 hours. After MGO removal, the rate of protein turnover was determined by examining TCA soluble radioactivity over time.

Figure 5: Model of AGE accumulation and proteopoise. Consumption of a high Gl diet causes enhanced oxidation and glycation induced protein damage. When levels of damaged proteins are low, the ubiquitin and the lysosomal/autophagic proteolytic systems can degrade the damaged proteins and toxicity is averted (top). Under chronic glycative stress glycated proteins accumulate. These may include AGEs along with unmodified proteins, some including ubiquitin conjugates (box). Some may oligomerize and crosslink forming the higher mass aggregates. Conjugates that are not degraded may also accumulate if there is insufficient proteasomal, including deubiquitinating, activity. Accumulated oligomerized altered proteins may impair the proteolytic machinery, setting up a vicious cycle of stress, limited proteolytic editing, and further damage to the proteome, resulting in some of the diseaserelated accumulation of AGEs and conjugates observed in vivo. 


\section{References:}

Biesemeier, A., Taubitz, T., Julien, S., Yoeruek, E., and Schraermeyer, U. (2014). Choriocapillaris breakdown precedes retinal degeneration in age-related macular degeneration. Neurobiology of aging 35, 2562-2573.

Bousova, I., Pruchova, Z., Trnkova, L., and Drsata, J. (2011). Comparison of glycation of glutathione Stransferase by methylglyoxal, glucose or fructose. Molecular and cellular biochemistry 357, 323-330. Buschhorn, B.A., and Peters, J.M. (2006). How APC/C orders destruction. Nat Cell Biol 8, 209-211. Cai, W., Uribarri, J., Zhu, L., Chen, X., Swamy, S., Zhao, Z., Grosjean, F., Simonaro, C., Kuchel, G.A., Schnaider-Beeri, M., et al. (2014). Oral glycotoxins are a modifiable cause of dementia and the metabolic syndrome in mice and humans. Proc Natl Acad Sci U S A 111, 4940-4945.

Chiu, C.-J., Liu, S., Willett, W. C., Wolever, T.M.S., Brand-Miller, J. C. Barclay, A. C., A. Taylor (2011). Informing food choices and health outcomes by use of the dietary glycemic index Nutrition Reviews 69, 231-242.

Chiu, C.J., Hubbard, L.D., Armstrong, J., Rogers, G., Jacques, P.F., Chylack, J., L. T., Hankinson, S.E., Willett, W.C., and Taylor, A. (2006a). Dietary glycemic index and carbohydrate in relation to early agerelated macular degeneration. Am J Clin Nutr 83, 880-886.

Chiu, C.J., Milton, R.C., Gensler, G., and Taylor, A. (2006b). Dietary carbohydrate intake and glycemic index in relation to cortical and nuclear lens opacities in the Age-Related Eye Disease Study. Am J Clin Nutr 83, 1177-1184.

Chiu, C.J., Milton, R.C., Gensler, G., and Taylor, A. (2007a). Association between dietary glycemic index and age-related macular degeneration in nondiabetic participants in the Age-Related Eye Disease Study. Am J Clin Nutr 86, 180-188.

Chiu, C.J., Milton, R.C., Klein, R., Gensler, G., and Taylor, A. (2007b). Dietary carbohydrate and the progression of age-related macular degeneration: a prospective study from the Age-Related Eye Disease Study. Am J Clin Nutr 86, 1210-1218.

Chiu, C.J., Milton, R.C., Klein, R., Gensler, G., and Taylor, A. (2007c). Dietary Glycemic Index Is Related to Progression of Age-Related Macular Degeneration. Investigative Ophthalmology \& Visual Science 48, 2101.

Chung, M., Ma, J., Patel, K., Berger, S., Lau, J., and Lichtenstein, A.H. (2014). Fructose, high-fructose corn syrup, sucrose, and nonalcoholic fatty liver disease or indexes of liver health: a systematic review and meta-analysis. Am J Clin Nutr 100, 833-849.

Colman, R.J., Anderson, R.M., Johnson, S.C., Kastman, E.K., Kosmatka, K.J., Beasley, T.M., Allison, D.B., Cruzen, C., Simmons, H.A., Kemnitz, J.W., et al. (2009). Caloric restriction delays disease onset and mortality in rhesus monkeys. Science 325, 201-204.

Cuervo, A.M., and Dice, J.F. (1998). How do intracellular proteolytic systems change with age? Front Biosci 3, d25-43.

Cuervo, A.M., and Dice, J.F. (2000). Age-related decline in chaperone-mediated autophagy. J Biol Chem 275, 31505-31513.

Dudek, E.J., Shang, F., Valverde, P., Liu, Q., Hobbs, M., and Taylor, A. (2005). Selectivity of the ubiquitin pathway for oxidatively modified proteins: relevance to protein precipitation diseases. Faseb J 19. Fritsche, L.G., Chen, W., Schu, M., Yaspan, B.L., Yu, Y., Thorleifsson, G., Zack, D.J., Arakawa, S., Cipriani, V., Ripke, S., et al. (2013). Seven new loci associated with age-related macular degeneration. Nat Genet 45, 433-439, 439e431-432.

Jenkins, D.J., Kendall, C.W., Augustin, L.S., Franceschi, S., Hamidi, M., Marchie, A., Jenkins, A.L., and Axelsen, M. (2002). Glycemic index: overview of implications in health and disease. Am J Clin Nutr 76, 266S-273S. 
Kaushik, S., Wang, J.J., Flood, V., Tan, J.S., Barclay, A.W., Wong, T.Y., Brand-Miller, J., and Mitchell, P. (2008). Dietary glycemic index and the risk of age-related macular degeneration. Am J Clin Nutr 88, 1104-1110.

Lim, J.S., Mietus-Snyder, M., Valente, A., Schwarz, J.M., and Lustig, R.H. (2010). The role of fructose in the pathogenesis of NAFLD and the metabolic syndrome. Nature reviews. Gastroenterology \& hepatology 7, 251-264.

Ludwig, D.S. (2002). The glycemic index: physiological mechanisms relating to obesity, diabetes, and cardiovascular disease. JAMA. 287, 2414-2423.

Lustig, R.H. (2013). Fructose: its " Alcohol without the buzz". Adv Nutr 4, 226-235.

Marques, C., Guo, W., Pereira, P., Taylor, A., Patterson, C., Evans, P.C., and Shang, F. (2006). The triage of damaged proteins: degradation by the ubiquitin-proteasome pathway or repair by molecular chaperones. Faseb J 20, 741-743.

Mattison, J.A., Roth, G.S., Beasley, T.M., Tilmont, E.M., Handy, A.M., Herbert, R.L., Longo, D.L., Allison, D.B., Young, J.E., Bryant, M., et al. (2012). Impact of caloric restriction on health and survival in rhesus monkeys from the NIA study. Nature 489, 318-321.

McCay, C., Crowell, M., and Maynard, L. (1935). The effect of retarded growth upon the length of life span and upon ultimate size. Journal of Nutrition 10, 63-79.

McCay, C.M., and Crowell, M.F. (1934). Prolonging the Life Span. The Scientific Monthly 39, 405-414. Morimoto, R.I., and Cuervo, A.M. (2014). Proteostasis and the aging proteome in health and disease. The journals of gerontology. Series A, Biological sciences and medical sciences 69 Suppl 1, S33-38. Mortimore, G.E., Lardeux, B.R., and Adams, C.E. (1988). Regulation of Microautophagyand Basal Protein Turnover in Rat Liver. J. Biol. Chem. 263, 2506-2512.

Park, C., and Cuervo, A.M. (2013). Selective autophagy: talking with the UPS. Cell biochemistry and biophysics 67, 3-13.

Pereira, P., Shang, F., Hobbs, M., Girao, H., and Taylor, A. (2003). Lens fibers have a fully functional ubiquitin-proteasome pathway. Exp Eye Res 76, 623-631.

Peters, J.M. (2002). The anaphase-promoting complex: proteolysis in mitosis and beyond. Mol Cell 9 , 931-943.

Pickart, C.M., and Fushman, D. (2004). Polyubiquitin chains: polymeric protein signals. Curr Opin Chem Biol 8, 610-616.

Rowan, S., Weikel, K., Chang, M.L., Nagel, B.A., Thinschmidt, J.S., Carey, A., Grant, M.B., Fliesler, S.J., Smith, D., and Taylor, A. (2014). Cfh genotype interacts with dietary glycemic index to modulate agerelated macular degeneration-like features in mice. Invest Ophthalmol Vis Sci 55, 492-501.

Sadowska-Bartosz, I., Galiniak, S., and Bartosz, G. (2014). Kinetics of glycoxidation of bovine serum albumin by glucose, fructose and ribose and its prevention by food components. Molecules 19, 1882818849.

Scribner, K.B., Pawlak, D.B., Aubin, C.M., Majzoub, J.A., and Ludwig, D.S. (2008). Long-term effects of dietary glycemic index on adiposity, energy metabolism, and physical activity in mice. Am J Physiol Endocrinol Metab 295, E1126-1131.

Semchyshyn, H.M., Miedzobrodzki, J., Bayliak, M.M., Lozinska, L.M., and Homza, B.V. (2014). Fructose compared with glucose is more a potent glycoxidation agent in vitro, but not under carbohydrateinduced stress in vivo: potential role of antioxidant and antiglycation enzymes. Carbohydrate research 384, 61-69.

Shang, F., Nowell, T.R., Jr., and Taylor, A. (2001). Removal of oxidatively damaged proteins from lens cells by the ubiquitin-proteasome pathway. Exp Eye Res 73, 229-238.

Smith, W., Assink, J., Klein, R., Mitchell, P., Klaver, C.C., Klein, B.E., Hofman, A., Jensen, S., Wang, J.J., and de Jong, P.T. (2001). Risk factors for age-related macular degeneration: Pooled findings from three continents. Ophthalmology. 108, 697-704. 
Taylor, A. (2012). Mechanistically linking age-related diseases and dietary carbohydrate via autophagy and the ubiquitin proteolytic systems. Autophagy 8, 1404-1406.

Tolkovsky, A.M. (2009). Mitophagy. Biochim Biophys Acta 1793, 1508-1515.

Uchiki, T., Weikel, K.A., Jiao, W., Shang, F., Caceres, A., Pawlak, D.B., Handa, J.T., Brownlee, M., Nagaraj, R., and Taylor, A. (2012). Glycation-altered proteolysis as a pathobiologic mechanism that links dietary glycemic index, aging, and age-related disease (in non diabetics). Aging Cell 11, 1-13.

Walford, R., and Crew, M. (1989). How Dietary Restriction Retards Aging: anIntegrative Hopothesis.

Growth, Develop. \& Aging 53, 139-140.

Walford, R.L. (1986). Caloric Restriction and Aging. C\&EN 11/10, 3.

Wang, T., Streeter, M.D., and Spiegel, D.A. (2015). Generation and characterization of antibodies against arginine-derived advanced glycation endproducts. Bioorganic \& medicinal chemistry letters.

Weikel, K., Taylor, A. (2011). Nutritional Modulation of Cataract and Age-Related Macular Degeneration. Mol Aspects Med 33, 318-375.

Weikel, K.A., Chiu, C.J., and Taylor, A. (2012a). Nutritional modulation of age-related macular degeneration. Mol Aspects Med 33, 318-375.

Weikel, K.A., Fitzgerald, P., Shang, F., Caceres, M.A., Bian, Q., Handa, J.T., Stitt, A.W., and Taylor, A. (2012b). Natural history of age-related retinal lesions that precede AMD in mice fed high or low glycemic index diets. Invest Ophthalmol Vis Sci 53, 622-632.

Weindruch, R. (1984). Dietary Restriction and the Aging Process. Free Radicals in Molecular Biology and Aging, preprint.

Weindruch, R. (1991). Retardation of aging by caloric restriction. In The potential for nutritional modulation of aging processes, D.K. Ingram, G.T. Baker III, and N.W. Shock, eds. (Trumbell, Connecticut: Food \& Nutrition Press, Inc.), pp. 109-121.

Weindruch, R. (1996). Caloric restriction and aging. Scientific American 274, 46-52.

Wong, E., Bejarano, E., Rakshit, M., Lee, K., Hanson, H.H., Zaarur, N., Phillips, G.R., Sherman, M.Y., and Cuervo, A.M. (2012). Molecular determinants of selective clearance of protein inclusions by autophagy. Nat Commun 3.

Yew, P.R. (2001). Ubiquitin-mediated proteolysis of vertebrate G1- and S-phase regulators. J Cell Physiol 187, 1-10.

Zhang, X., Dudek, E.J., Liu, B., Ding, L., Fernandes, A.F., Liang, J.J., Horwitz, J., Taylor, A., and Shang, F. (2007). Degradation of C-terminal Truncated \{alpha\}A-crystallins by the Ubiquitin Proteasome Pathway. Invest Ophthalmol Vis Sci 48, 4200-4208. 
Fig. 1

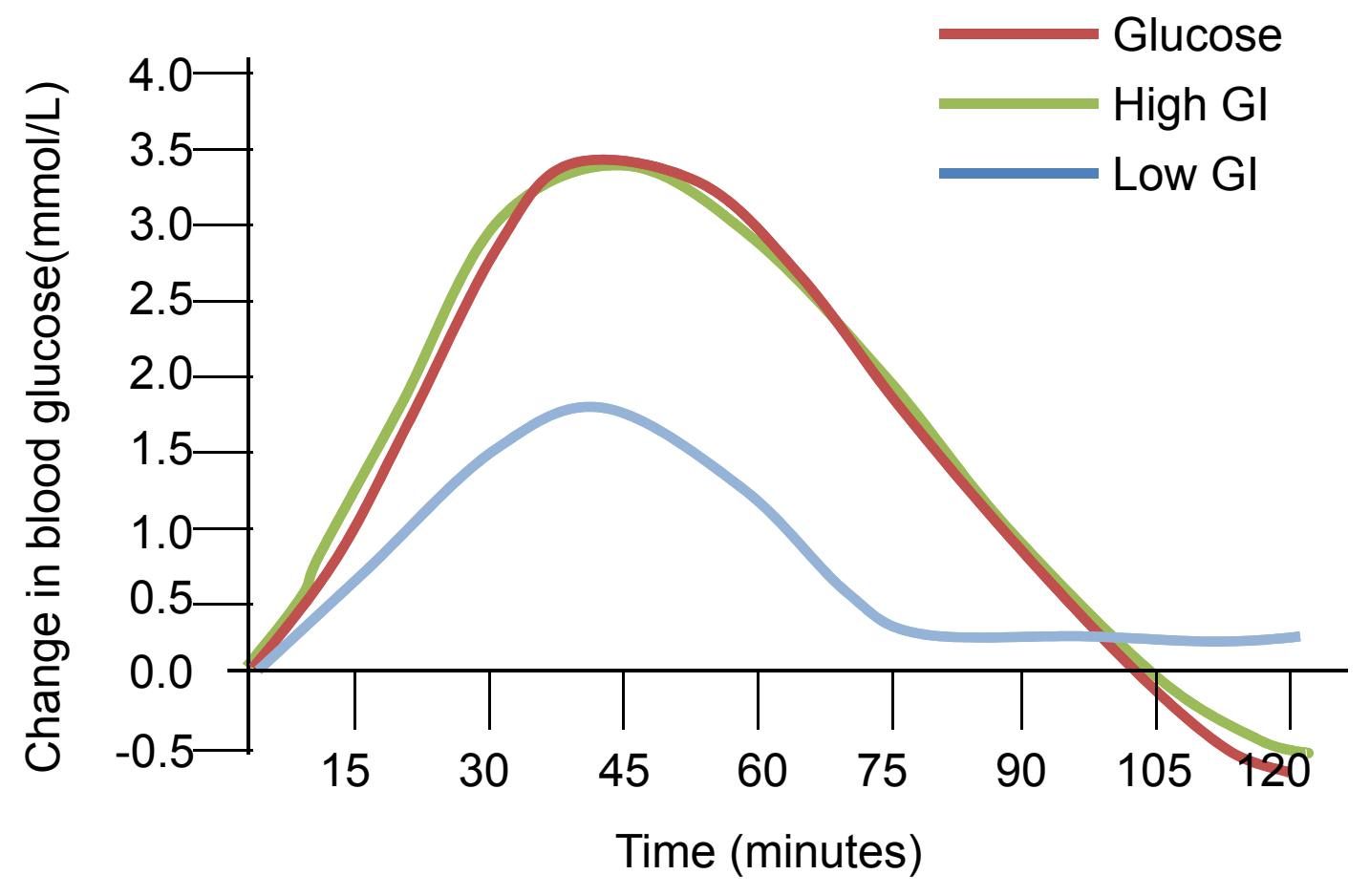


Fig. 2

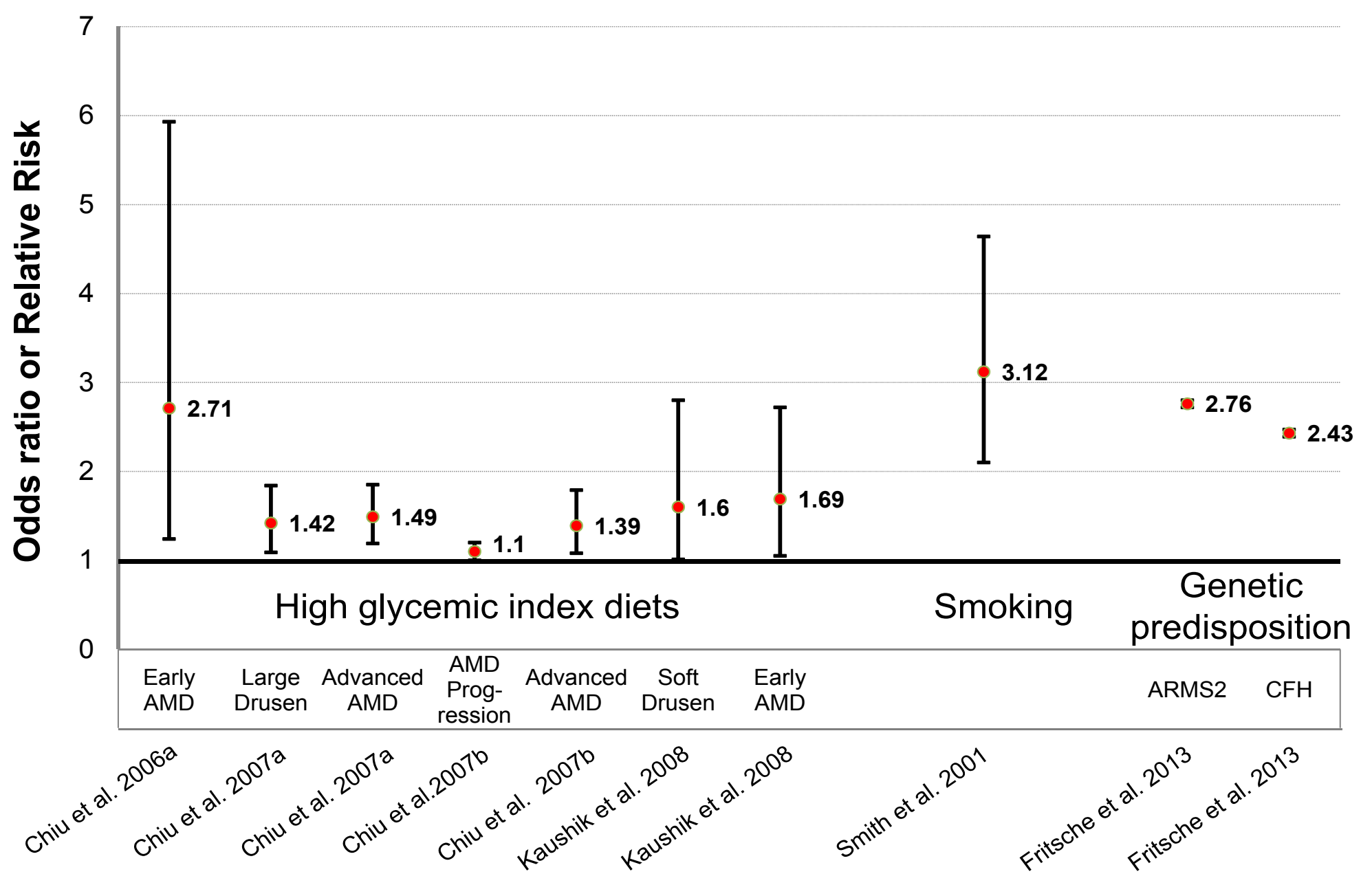


Fig. 3
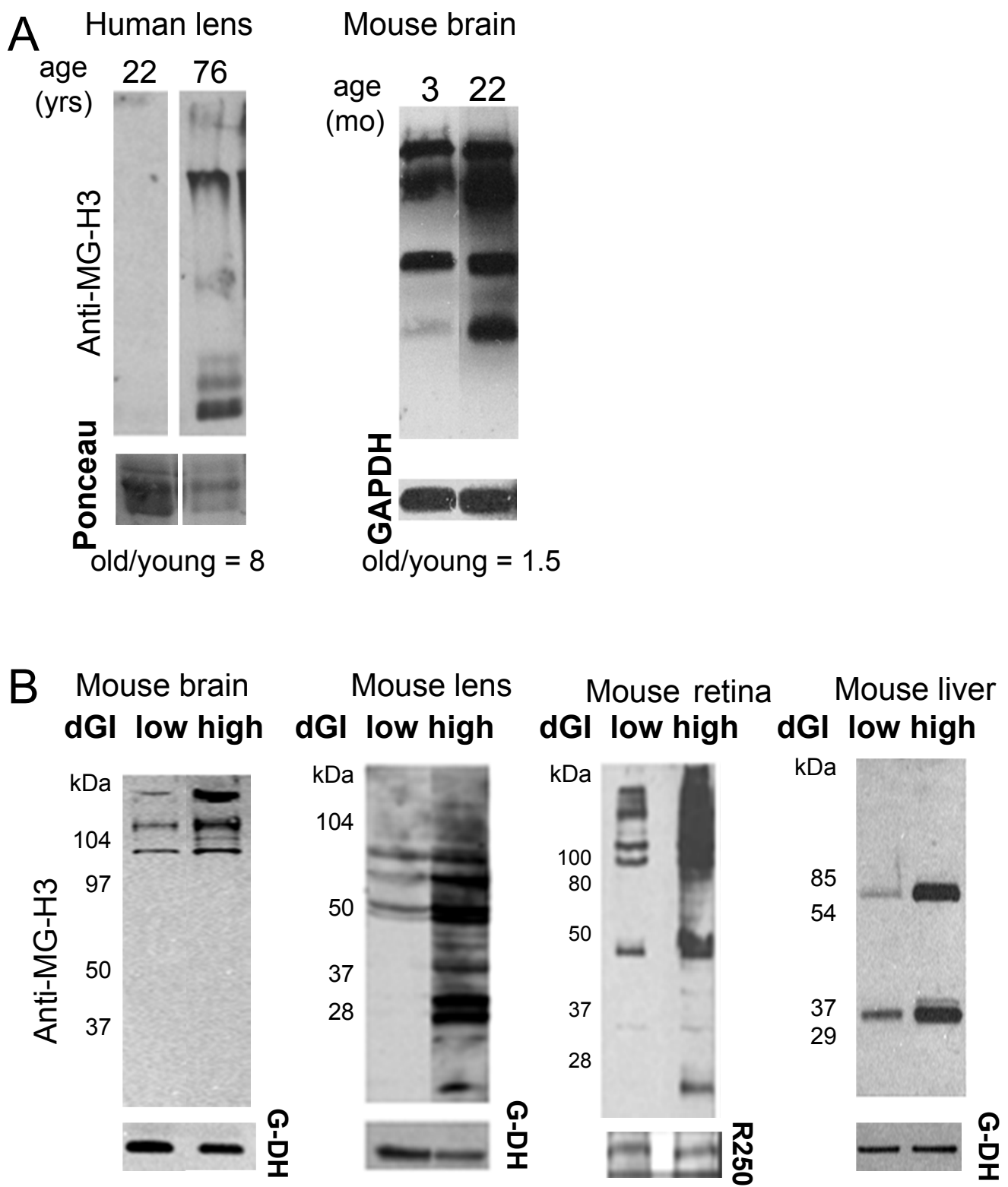

high/low = 7.5 high/low $=33.7 \quad$ high $/$ low $=3.0 \quad$ high $/$ low $=6.6$ 
Fig. 4

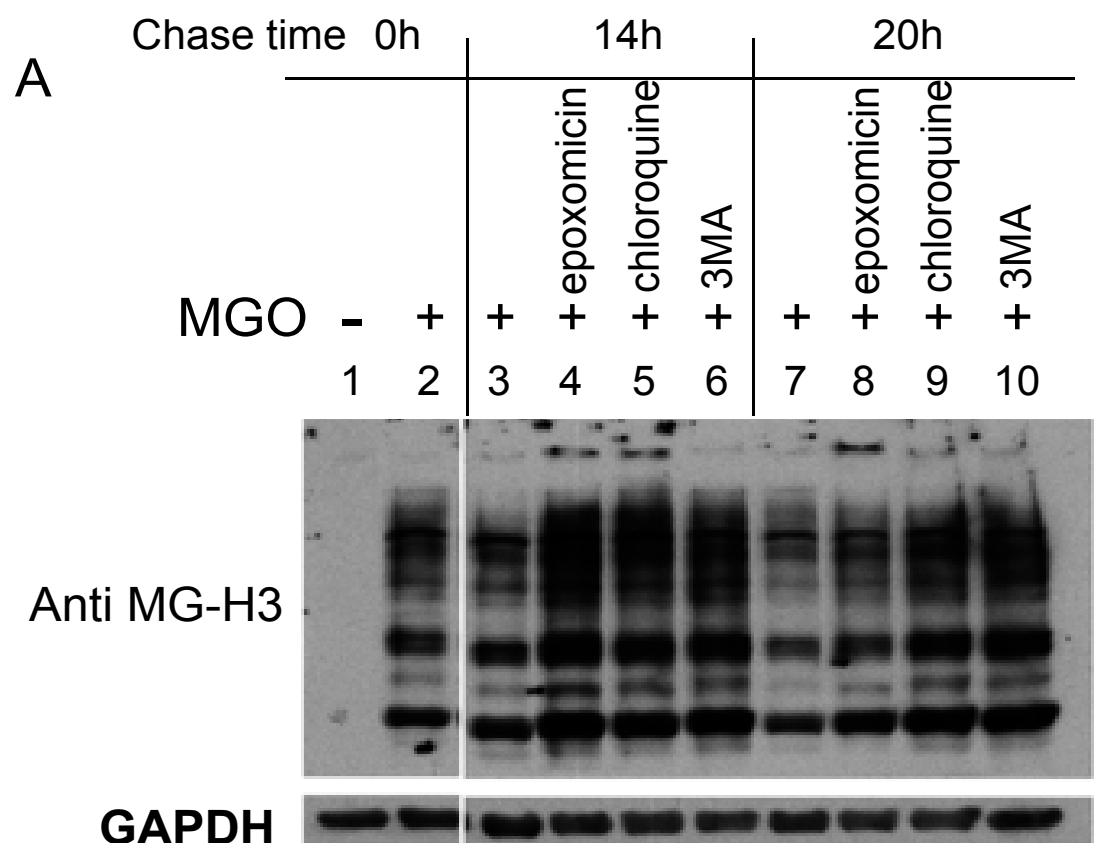

B

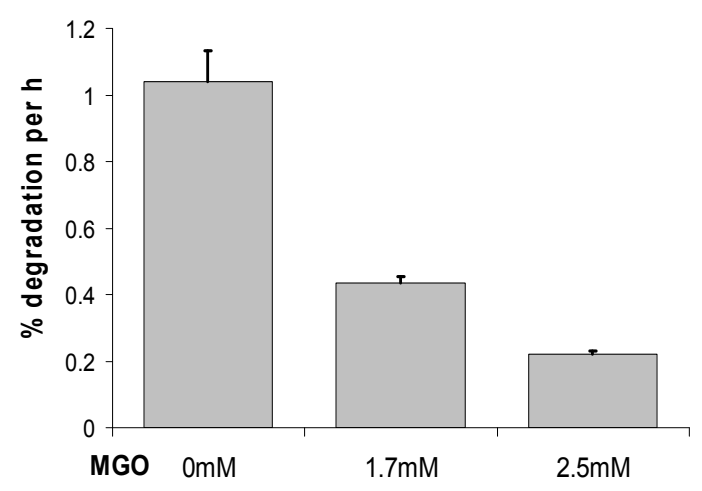

$\begin{array}{lllllllllll}\text { Relative AGE level } & 0 & 1 & 0.8 & 1.5 & 1.5 & 1.6 & 0.7 & 1.2 & 1.4 & 1.2\end{array}$ 
Fig. 5

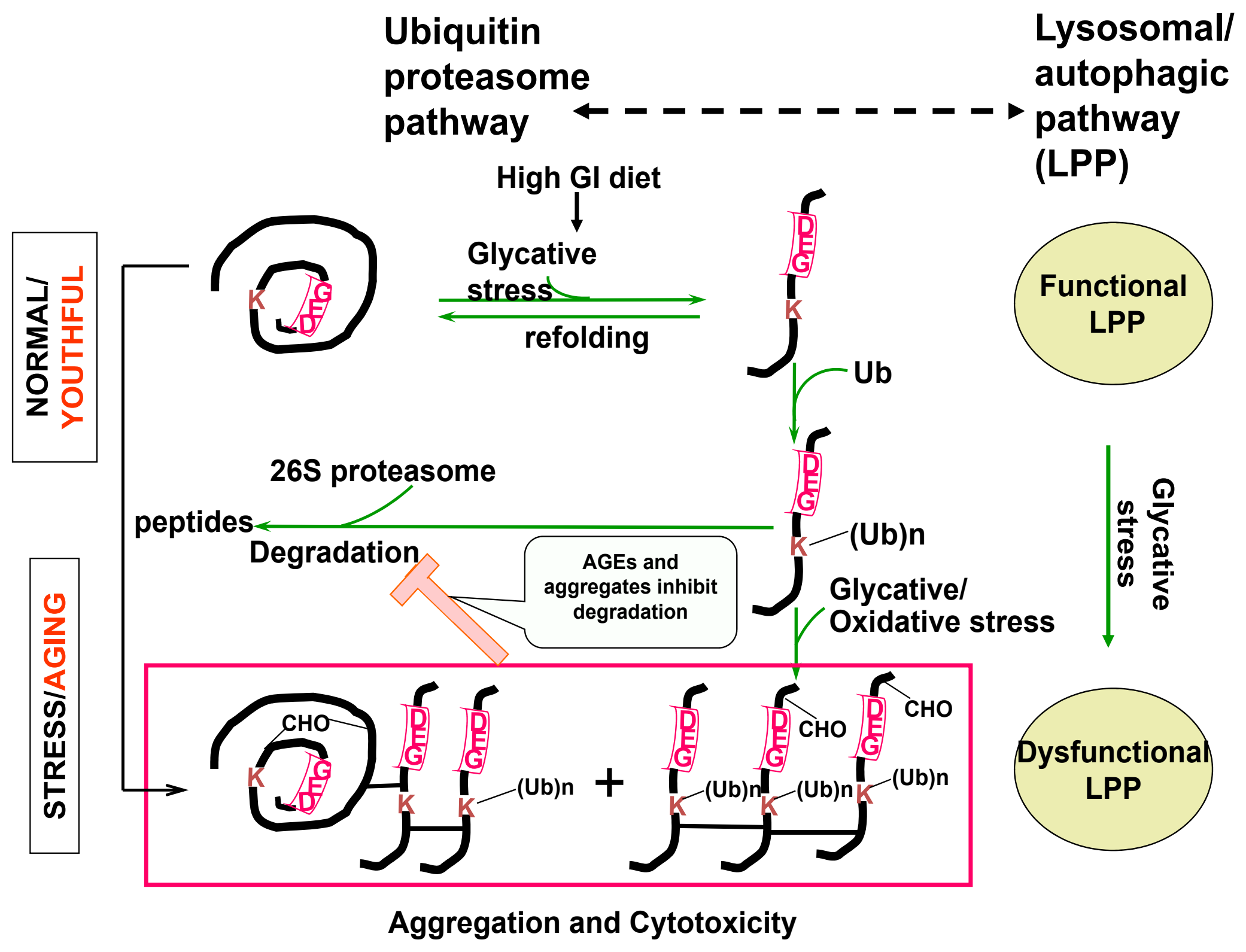

